New Perspectives on Solar Prominences, LUU Colloquium 167

ASP Conference Series, Vol. 150, 1998

David Webb, David Rust, and Brigitte Schmieder (eds.)

\title{
NLTE Model of a Quiescent Filament
}

\author{
E. V. Kononovich, A. B. Gorshkov, O. B. Smirnova \\ Sternberg Astronomical Institute, 119899 Moscow, Russia
}

P. Kotrč

Astronomical Institute, 25165 Ondřejov, Czech Republic

\begin{abstract}
The Sacramento Peak Observatory VTT Echelle Spectrograph was used to obtain high resolution spectrograms of the filament at W06 ${ }^{\circ} \mathrm{S} 27^{\circ}$ observed on April 9, 1991 in hydrogen $\mathrm{H} \alpha, \mathrm{H} \beta$, and CaII $\mathrm{H}$ and $\lambda 8498$ lines. The line profiles were measured and digitized. A numerical code based on the MALI approach with partial frequency redistribution (PRD) in resonance lines was applied to obtain theoretical profiles of hydrogen and CaII lines. As a result of fitting the observed and calculated profiles, the physical parameters of the filament plasma were obtained. They include temperature, gas pressure, electron density and turbulent velocity.
\end{abstract}

\section{Introduction}

In spite of the existence of a large number of treatments of the spectral line transfer problem for the solar chromosphere and prominences, a variety of improved observations stimulates further refinement of the existing models and codes. The first NLTE prominence models were of a one-dimensional (1D) slab type (Jefferies 1955, Jakovkin and Zeldina 1964). Sobolev (1962) considered a much more difficult case of a spherical configuration. The whole 1D problem has been fully examined during the last few decades. However, before 1985 mostly the complete frequency redistribution approximation (CRD) was used and even rather sophisticated multi-slab models were elaborated (for references see Heinzel et al. 1987). In their paper a large set of $1 \mathrm{D}$ models for the hydrogen atom with standard PRD lines were presented. The basic ideas of NLTE prominence modeling were summarized by Heinzel (1991) together with various departures from classical finite 1D homogeneous slab models.

All these treatments are restricted to the case of only one chemical element - presumably hydrogen (e.g., Heinzel et al. 1987) or CaII (e.g., Gouttebroze et al. 1997). On the other hand, it is necessary to include several elements to make the prominence plasma diagnostics less ambiguous. In this work the NLTE PRD code similar to that described by Heinzel (1995) is adopted for simultaneous analysis of hydrogen and Ca II lines in filaments observed on the solar disk, in contrast to the usual interpretation of prominence observations on the solar limb. The validity of such an approach was demonstrated in our previous analysis of the July 6 filament (Kotrč et al. 1994) by means of the cloud 


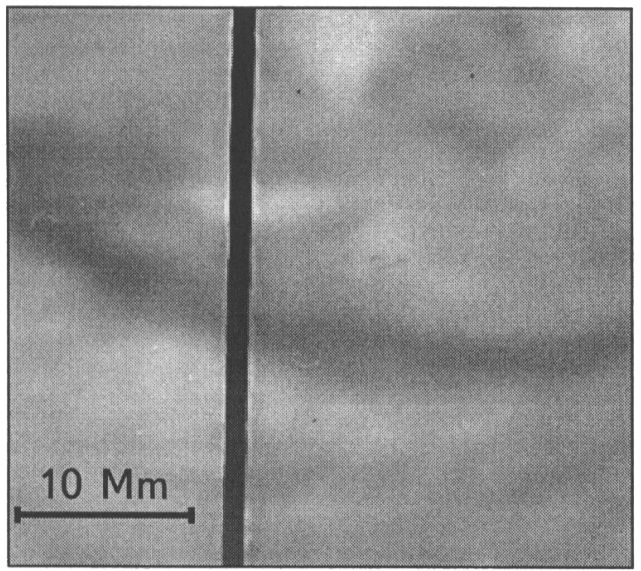

Figure 1. The H $\alpha$ slit-jaw picture of the Sac Peak Echelle Vacuum Spectrograph with filament $\mathrm{W} 06^{\circ} \mathrm{S} 27^{\circ}$ on April 9, 1991.

model applied to hydrogen and Ca II lines.

\section{Observations}

In this work we use the high resolution spectrograms of a quiescent filament obtained on April 9, 1991 under excellent atmospheric conditions. The observations were performed by one of us (P. Kotrč) at Sacramento Peak Observatory, operated by NSO, and the parameters of the VTT Echelle Spectrograph were given by Kotrc et al. (1994). The filament under investigation was at $\mathrm{W}^{\circ} 6^{\circ}$ $\mathrm{S} 27^{\circ}$, i.e., at the distance about $30^{\circ}$ from the solar disc center. Figure 1 presents the $\mathrm{H} \alpha$ slit-jaw image. The dark vertical line is the position of the spectrograph entrance slit. The hydrogen $\mathrm{H} \alpha, \mathrm{H} \beta, \mathrm{Ca}$ II H and $\lambda 8498$ lines were chosen for further analysis.

Accurate microphotometry was performed at Sternberg Astronomical Institute of Moscow State University. The digital AMD-1 densitometer was used. The photometric slit was of $50 \mu \times 50 \mu$ size. The scanning step was the same $(50 \mu)$, i.e., one step (pixel) corresponds to the distance of $126.5 \mathrm{~km}$ or $0.175 \mathrm{arc}$ sec on the Sun. The photometric calibration was done by fitting the reference profiles to the standard profiles for the quiet Sun (Kurucz 1990).

Figure 2 shows the obtained photometric cross sections of the filament (i.e., mean profile along the slit) for all considered lines, and Figure 3 presents spectral line profiles for 5 distances from the filament channel. The central line intensities are given in Table 1.

\section{Modeling}

The filament line profiles definitely differ from the undisturbed chromospheric profile as is seen in Figure 4. The difference is not large and it is smaller for more transparent lines. This demands a higher accuracy of photometry and model 


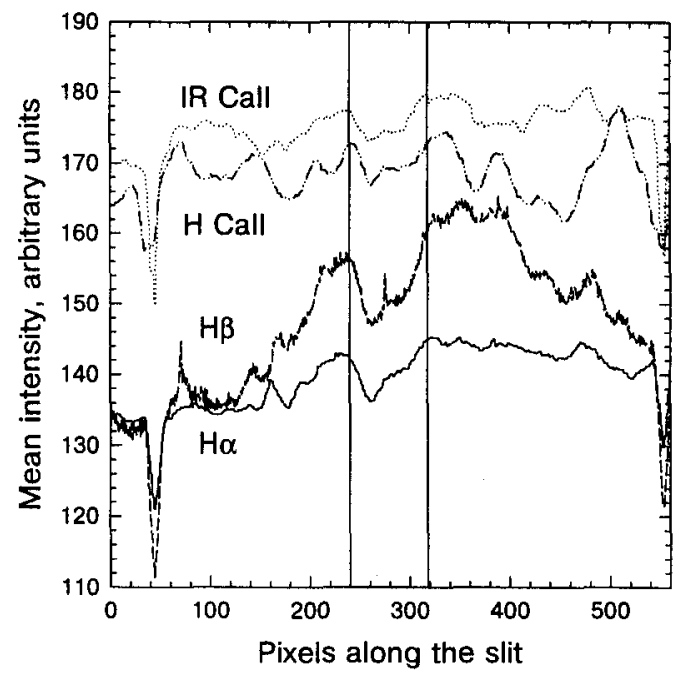

Figure 2. Photometric profiles of the April 9, 1991 filament averaged over the central parts of the corresponding lines. The filament is between the two vertical lines.

Table 1. Central line intensities in \% of the nearby continuum

\begin{tabular}{lcc}
\hline Line & Quiet Sun & Filament Channel \\
\hline $\mathrm{H} \alpha$ & 16 & 12 \\
$\mathrm{H} \beta$ & 14 & 6 \\
$\mathrm{CaII} \mathrm{H}$ & 5 & 2 \\
$\mathrm{CaII} \lambda 8498$ & 28 & 18 \\
\hline \hline
\end{tabular}

Table 2. The Filament Model

\begin{tabular}{lcc}
\hline$T=9500 K$ & $P=0.13 \mathrm{din} / \mathrm{cm}^{3}$ & $V_{\text {turb }}=8 \mathrm{~km} / \mathrm{s}$ \\
\hline \multicolumn{3}{c}{} \\
\hline \hline Line & \multicolumn{1}{c}{$\tau_{0}$} & $J, 10^{6} \mathrm{erg} /\left(\mathrm{s} \cdot \mathrm{ster} \cdot \mathrm{cm}^{2}\right)$ \\
\hline $\mathrm{H} \alpha$ & 7.8 & 6.1 \\
$\mathrm{H} \beta$ & 1.1 & 4.4 \\
CaII H & 20.1 & 1.3 \\
CaII $\lambda 8498$ & 0.1 & 26.6 \\
\hline \hline
\end{tabular}



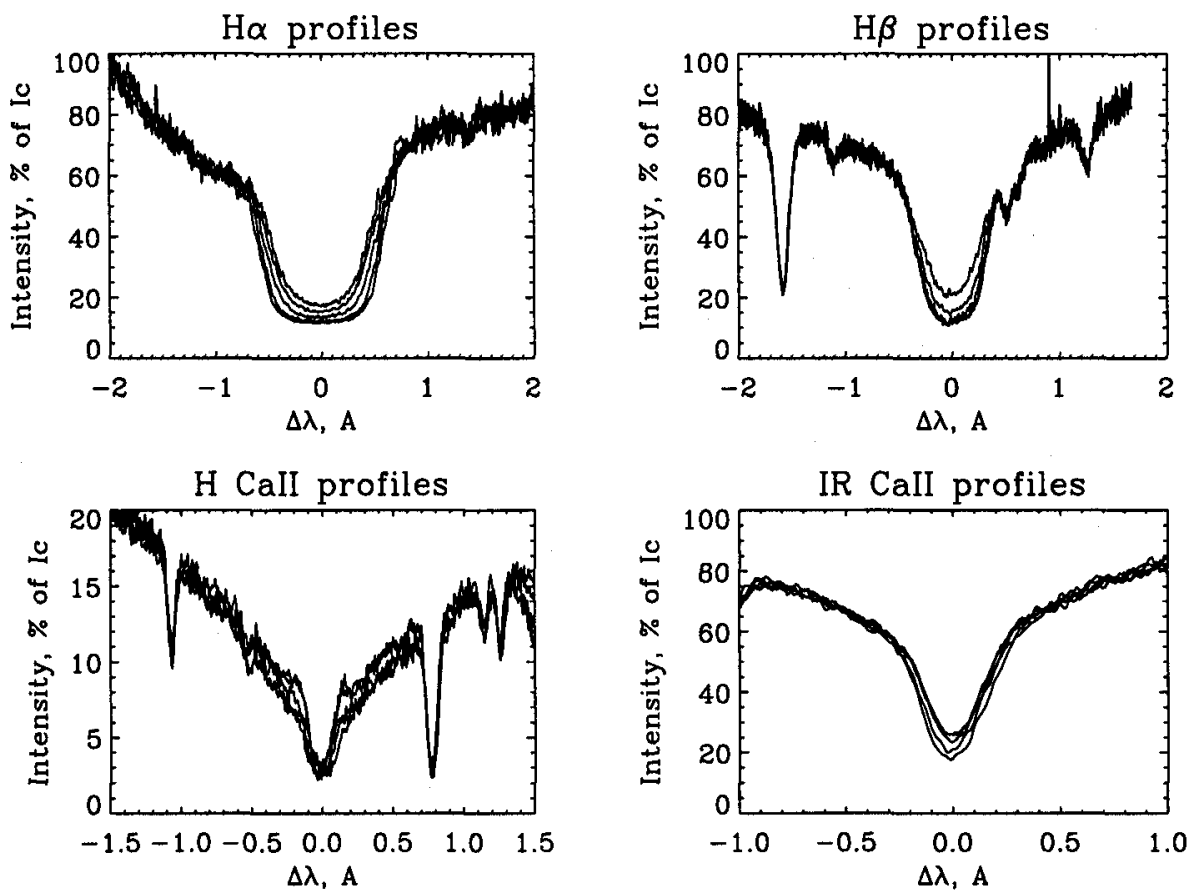

Figure 3. Line profiles at different points along the slit: a) $\mathrm{H} \alpha, \mathrm{b}$ ) $\mathrm{H} \beta$, c) $\mathrm{H} \mathrm{CaII}$, and d) $\lambda 8498 \mathrm{CaII}$, in \% of the nearby continuum.

calculations. Nevertheless, even the cloud model used in our previous treatment (Kotrč et al. 1994) has led to a rather good agreement between observed and calculated intensities. In this work we apply the 1D NLTE PRD code. It is similar to that described by Heinzel (1995) but adopted to simultaneous analyses of $\mathrm{H}$ and $\mathrm{Ca}$ II lines. The model parameters were searched using the method of trial and error beginning from the hydrogen lines to obtain a value of the electron density. Then the calcium lines were calculated.

The resulting filament model is summarized in Table 2 with temperature $T=9500 \mathrm{~K}, P=0.13 \mathrm{din} / \mathrm{cm}^{3}, V_{t u r b}=8 \mathrm{~km} / \mathrm{s}$ and electron density in the filament center $n_{e}=4.2 \cdot 10^{10} \mathrm{~cm}^{-3}$.

\section{Discussion}

For the first time we present results concerning the PRD transfer problem solution for the case of joint spectroscopy of two different atomic species, namely, hydrogen and $\mathrm{Ca}$ II. The procedure was applied to the filament spectral lines $\lambda 6563,4861,3968,8498$ of both of these elements. The resulting physical parameters, $T, P, P_{e}$, and $V_{t u r b}$, obtained by fitting the observed and calculated line profiles are in good agreement with other prominence models. The accuracy of the fitting procedure for the four lines varies from 1 to $5 \%$. The best fit is for the $\mathrm{H} \beta$ and $\mathrm{Ca}$ II $\mathrm{H}$ lines. We suggest that such a good agreement for both lines 

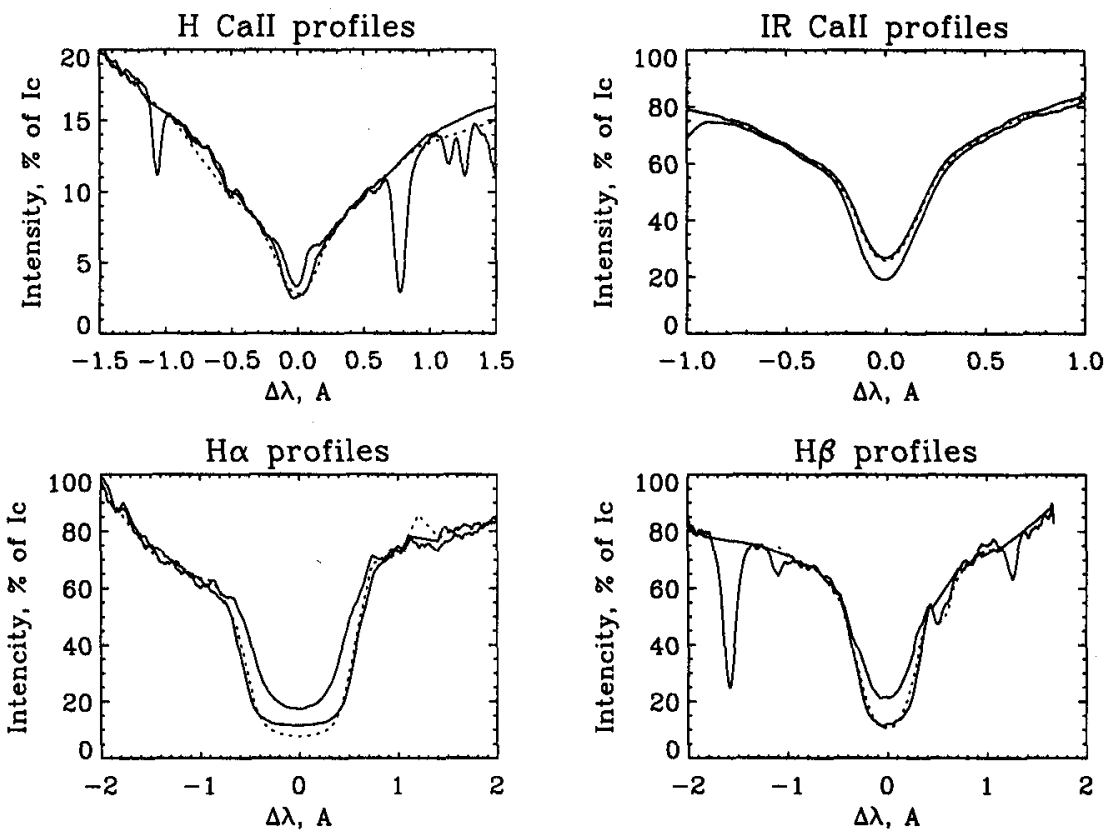

Figure 4. Calculated line profiles (dashed) in comparison to the observed ones (lower full lines). Upper full lines are the incident radiation profiles used in the calculations.

is due to their intermediate value of the corresponding total optical thickness in the line center $1<\tau_{c}<10$. That means that the observed emission originates along the whole filament thickness. Concerning the optically thick $\mathrm{H} \alpha$ line one may suggest the existence of some unconsidered transition process in the upper border of the filament, where the line core is forming.

We still do not understand the large discrepancy for the infrared Ca II line. Cloud model calculations led to a rather small ratio $(\sim 5$, see Kotrč et al. 1994) of optical depths between the resonance $H$ line and subordinate $\lambda 8498$ line. Meanwhile NLTE calculations gave a much larger value, $\sim 200$. This contradicts the qualitative result that the filament is clearly seen on the original $\lambda 8498$ spectrogram; according to the model calculation it is not expected to be seen. A possible explanation may be found in terms of inhomogenious structure of the filament consisting of "hot" and "cold" small fibrills. This may be possible if the IR calcium line source function depends mostly on the temperature values, but not on the density.

\section{References}

Gouttebroze, P., Vial, J.-C. and Heinzel, P. 1997, Sol. Phys., 172, 125

Heinzel, P., Gouttebroze, P. and Vial, J.-C. 1987, A\&A, 183, 351 
Heinzel, P. 1991, Preprint 122, Astron. Inst. Czechosl. Acad. Sci.

Heinzel, P. 1995, A\&A, 299, 563

Jakovkin, N. A. and Zeldina, M. Yu. 1964, AZh, 41, 914 (Russian)

Jefferies, J. T. 1955, MNRAS, 115, 617

Kotrč, P., Heinzel, P., Gorshkov, A. B., Kononovich, E. V., Kupryakov, Yu. A. and Smirnova, O. B. 1994, in Solar Coronal Structures, (eds.) V. Rusin, P. Heinzel and J.-C. Vial, VEDA Publ. Comp., Bratislava, p. 361

Kurucz, R. L. 1990, in The Solar Interior and Atmosphere, (eds.) A. N. Cox, W. C. Livingston and M. Matthews, Univ. of Arizona Press, Tucson, AZ, p. 663

Sobolev V. V. 1962, AZh, 39, 632 (Russian) 